\title{
Long-Term Clinical Course in a Patient with Complete Congenital Stationary Night Blindness
}

\author{
Kentaro Kurata Katsuhiro Hosono Yoshihiro Hotta \\ Department of Ophthalmology, Hamamatsu University School of Medicine, \\ Hamamatsu, Japan
}

\section{Keywords}

Complete congenital stationary night blindness - Long-term observation - Visual function .

Electroretinogram

\begin{abstract}
Background: This report describes a 45-year-old man with complete congenital stationary night blindness (CSNB1) who has been followed up for 38 years. Case: The patient first visited our hospital as a 7-year-old boy with a complaint of low visual acuity. Best corrected visual acuity (BCVA) was 0.5 in the right eye and 0.6 in the left eye. The refractive error was approximately $-5.0 \mathrm{D}$ in both eyes. The fundus showed only myopic changes. A bright-flash electroretinogram (ERG) revealed a negative configuration. We diagnosed CSNB and corrected the refractive error with glasses. We continued to monitor the ERG and various waveform components as well as visual acuity and the appearance of the fundus. All NYX exons were screened for a causative mutation by polymerase chain reaction amplification, and direct sequencing was performed. Results: By 10 years of age, BCVA had increased to 0.8 on the right and 0.9 on the left, with little change thereafter. The fundus continued to show only myopic changes. No changes were seen in the amplitude or implicit time of the a-wave or b-wave or in the b/a-wave ratio. A novel hemizygous insertion mutation, c.1205_1206insT, p.(Glu404Argfs*89), was detected in exon 2 of the NYX gene. Conclusion: To our knowledge, this is the longest follow-up of a patient with CSNB1. No changes in the clinical course have been seen during follow-up. We believe that it is important to continue observations and accumulate clinical data for prognostic purposes on patients with CSNB1.
\end{abstract}

(C) 2017 The Author(s)

Published by S. Karger AG, Basel 


\section{Introduction}

The Schubert-Bornschein type of congenital stationary night blindness (CSNB) is a nonprogressive retinal disorder characterized by impaired dark adaptation, decreased visual acuity, refractive error, nystagmus, and strabismus. The clinical characteristics of CSNB include an essentially normal fundus except for myopic changes, such as tilted optic discs with temporal pallor. The bright-flash, mixed rod-cone electroretinogram (ERG) in a patient with CSNB has a negative configuration, i.e., the b-wave is smaller than the a-wave [1].

Miyake et al. [2, 3] classified CSNB into complete CSNB (CSNB1) and incomplete CSNB (CSNB2), and demonstrated that these 2 entities are physiologically independent diseases. According to their research, CSNB1 is characterized by "on" bipolar pathway dysfunction and CSNB2 is characterized by "on" and "off" bipolar pathway dysfunction. Patients with CSNB1 show no detectable rod response on the ERG, whereas CSNB2 patients show a reduced but recordable rod response.

CSNB segregates into X-linked recessive and autosomal recessive forms. To date, CSNB1 and CSNB2 have been associated with 5 and 2 causative genes, respectively [4]. NYX is the most common causative gene in CSNB1, whereas CACNA1F is the most common in CSNB2 [5]. Recently, GNB3 was reported to be a novel causative gene [6].

CSNB is considered to be non-progressive, but to our knowledge there are few reports based on long-term observation. In this report, we describe the outcome in a patient with CSNB1 whom we have followed up for over 38 years.

\section{Case Report}

The patient first visited Hamamatsu University Hospital as a 7-year-old boy in January 1978 with a complaint of low visual acuity. He had previously been diagnosed with CSNB at the Department of Ophthalmology in Nagoya University Hospital at the age of 4 years. He complained of night blindness but had no family history of retinal disease.

\section{Ocular Findings on Initial Evaluation}

On initial examination, the patient's best corrected decimal acuity was 0.5 in the right eye and 0.6 in the left eye. The refractive error was $-6.0 \mathrm{D}$ in the right eye and $-5.25 \mathrm{D}$ in the left eye. The anterior segments were normal, and no nystagmus or strabismus was found. The appearance of the fundus was essentially normal, except for myopic changes. The visual fields were normal on Goldmann perimetry. A dark adaptation curve from a GoldmannWeekers adaptometer showed an absence of rod adaptation. The bright-flash, mixed rodcone ERG elicited by a 20-J white flash stimulus after $20 \mathrm{~min}$ of dark adaptation showed a normal a-wave amplitude but a significantly reduced b-wave amplitude (a so-called "negative configuration"). The oscillatory potential was unremarkable (Fig. 1a). A diagnosis of CSNB was confirmed. The refractive error was corrected by glasses.

\section{Evaluation of the Clinical Course}

Best corrected visual acuity (BCVA) and funduscopy were monitored frequently. We performed a mixed rod-cone ERG each year under the same conditions as those at the initial evaluation until the patient reached the age of 26 years, when he stopped attending for follow-up. At the age of 34 years, the patient resumed attending the hospital. By this time, the instrument we used to perform the mixed rod-cone ERG had been replaced, so the ERG was 
performed under changed conditions, i.e., elicited by a $200 \mathrm{~cd} \times \mathrm{s} / \mathrm{m}^{2}$ white flash stimulus after 20 min of dark adaptation. The various components of these waveforms were analysed.

\section{Findings during the Clinical Course}

By 10 years of age, BCVA had increased to 0.8 in the right eye and 0.9 in the left eye, and has remained unchanged thereafter (Fig. 2a). The myopia has progressed (Fig. 2b). The data for the various components of the mixed rod-cone ERG are shown in Figure 2c-g. Although it was not possible to compare the data obtained using the different instruments, no changes could be seen in the amplitudes or implicit times of the a-wave and b-wave or in the b/awave ratio.

When the patient was 38 years of age, full-field ERGs were performed according to the International Society for Clinical Electrophysiology of Vision protocol [7]. The scotopic 0.01 ERG after 20 min of dark adaptation was non-recordable. However, the photopic 3.0 ERG and the $30-\mathrm{Hz}$ flicker with a background light intensity of $25 \mathrm{~cd} / \mathrm{m}^{2}$ after $10 \mathrm{~min}$ of light adaptation were within normal limits (Fig. 1b). On the basis of these ERG findings, we made a diagnosis of CSNB1.

The patient continues to be followed up as an outpatient at our hospital. BCVA is presently 0.8 in the right eye and 0.8 in the left eye. The refractive error is $-14.5 \mathrm{D}$ in the right eye and $-14.75 \mathrm{D}$ in the left eye. The fundus shows myopic changes but no atrophic changes (Fig. 3). Visual fields are normal on Goldmann perimetry. Central retinal thickness and retinal lamination are normal on spectral-domain optical coherence tomography (Fig. 3).

\section{DNA Analysis}

Written informed consent was obtained from the patient before the molecular genetic study. Genomic DNA was extracted from leucocytes in peripheral blood. Both exons of the NYX gene, including the coding regions and intron-exon boundaries, were amplified by PCR (polymerase chain reaction), and direct sequencing was performed. The PCR and sequencing procedures used have been described previously [8]. The primer sequences are available on request. Nucleotide numbering reflects cDNA numbering with +1 corresponding to the $\mathrm{A}$ of the ATG translation initiation codon in the reference sequence NM_022567.2. Consequently, the patient was detected to have a novel hemizygous insertion mutation, c.1205_1206insT, p.(Glu404Argfs*89), in exon 2 of the $N Y X$ gene. This mutation did not appear in the public single nucleotide polymorphism databases (1,000 Genomes database [http://www. 1000genomes.org/], Exome Aggregation Consortium database [http://exac.broadinstitute. org/], and Human Genetic Variation Database [http://www.hgvd.genome.med.kyoto-u.ac.jp/ index.html], all accessed on November 11, 2016).

\section{Discussion}

CSNB can be classified into CSNB1 or CSNB2 based on ERG findings [2]. We diagnosed CSNB1 in our patient on the basis of failure of rod system function in the presence of essentially normal cone system reactions. In addition, we identified a novel hemizygous insertion mutation, c.1205_1206insT, p.(Glu404Argfs*89), in NYX, which is one of the causative genes of CSNB1.

It is generally believed that the clinical course in patients with CSNB1 does not change over time. However, to our knowledge, there are few reports in which a patient has been observed for longer than 10 years [9], so doubts remain as to whether CSNB1 can be defined 
as truly non-progressive. We followed up our patient for 38 years. Compared to other reports of CSNB1 cases, our study has the longest follow-up duration.

We frequently performed a mixed rod-cone ERG examination in this patient between the ages of 7 and 26 years, and again from the age of 34 years to the present; the examination during these 2 periods was performed under different conditions. No changes were seen in the amplitude or implicit time of either the a-wave or b-wave after analysing the waveform results. Weleber [10] reported that the amplitude of the b-wave in a mixed rod-cone ERG for normal subjects was affected by age, whereas the implicit times of the a-wave and bwave and the amplitude of the a-wave were not affected. According to the data reported, the amplitude of the b-wave is preserved up to the 3rd decade of life and decreases from the 4th decade onwards. However, no change was observed in the amplitude of the b-wave on the mixed rod-cone ERG in our patient, even after the age of 40 years. Given that the "on" bipolar pathways fail completely in patients with CSNB1, the b-wave for the mixed rod-cone response mainly reflects the functions of cone "off" bipolar cells in these patients. Therefore, although the functions of rod and cone bipolar cells in normal individuals are thought to diminish with age, it is possible that the functions of the cone "off" bipolar cells in patients with CSNB1 are not affected by age. It is important to follow the course of these patients and measure their b-wave amplitudes continuously.

Abnormalities of the fundus other than myopic changes are generally not seen in patients with CSNB. Apart from myopic changes, our patient continues to have a normal fundus. BCVA was improved by correcting the existing refractive error with glasses, and hardly any changes occurred thereafter. However, there are a few reports of atypical cases of vision loss progressing alongside the appearance of retinal and optic disc atrophy in patients with CSNB2 caused by CACNAF1 mutations [11, 12].

In conclusion, we have followed up a patient with CSNB1 for 38 years and found no changes in the clinical course of the disease during this time. Accumulation of further clinical data by ongoing observation of this patient's progress in the future will not only provide useful information about the clinical course of the condition but also assist with predicting the prognosis in patients with CSNB1.

\section{Statement of Ethics}

This research was approved by the Institutional Review Board at Hamamatsu University School of Medicine and conformed to the tenets of the Declaration of Helsinki. Written informed consent to publish was obtained from the patient reported on here.

\section{Disclosure Statement}

The authors declare that they have no conflicts of interest related to this manuscript. 


\section{Case Reports in Ophthalmology}

\begin{tabular}{l|l}
\hline Case Rep Ophthalmol 2017;8:237-244 \\
\hline DOI: $10.1159 / 000462961$ & $\begin{array}{l}\text { (c) } 2017 \text { The Author(s). Published by S. Karger AG, Basel } \\
\text { www.karger.com/cop }\end{array}$ \\
\hline
\end{tabular}

Kurata et al.: Long-Term Clinical Course in a Patient with Complete Congenital Stationary Night Blindness

\section{References}

1 Schubert G, Bornschein H: Analysis of the human electroretinogram (in German). Ophthalmologica 1952;123:396-413.

2 Miyake Y, Yagasaki K, Horiguchi M, Kawase Y, Kanda T: Congenital stationary night blindness with negative electroretinogram. A new classification. Arch Ophthalmol 1986;104:1013-1020.

-3 Miyake Y, Yagasaki K, Horiguchi M, Kawase Y: On- and off-responses in photopic electroretinogram in complete and incomplete types of congenital stationary night blindness. Jpn J Ophthalmol 1987;31:8187.

4 Zeitz C, Robson AG, Audo I: Congenital stationary night blindness: an analysis and update of genotypephenotype correlations and pathogenic mechanisms. Prog Retin Eye Res 2015;45:58-110.

-5 Bijveld MM, Florijn RJ, Bergen AA, van den Born LI, Kamermans M, Prick L, Riemslag FC, van Schooneveld MJ, Kappers AM, van Genderen MM: Genotype and phenotype of 101 Dutch patients with congenital stationary night blindness. Ophthalmology 2013;120:2072-2081.

-6 Vincent A, Audo I, Tavares E, Maynes JT, Tumber A, Wright T, Li S, Michiels C; GNB3 Consortium, Condroyer C, MacDonald H, Verdet R, Sahel JA, Hamel CP, Zeitz C, Héon E: Biallelic mutations in GNB3 cause a unique form of autosomal-recessive congenital stationary night blindness. Am J Hum Genet 2016;98:1011-1019.

7 Marmor MF, Holder GE, Seeliger MW, Yamamoto S; International Society for Clinical Electrophysiology of Vision: Standard for clinical electroretinography (2004 update). Doc Ophthalmol 2004;108:107-114

-8 Hosono K, Ishigami C, Takahashi M, Park DH, Hirami Y, Nakanishi H, Ueno S, Yokoi T, Hikoya A, Fujita T, Zhao Y, Nishina S, Shin JP, Kim IT, Yamamoto S, Azuma N, Terasaki H, Sato M, Kondo M, Minoshima S, Hotta Y: Two novel mutations in the EYS gene are possible major causes of autosomal recessive retinitis pigmentosa in the Japanese population. PLoS One 2012;7:e31036.

-9 Dryja TP, McGee TL, Berson EL, Fishman GA, Sandberg MA, Alexander KR, Derlacki DJ, Rajagopalan AS: Night blindness and abnormal cone electroretinogram $\mathrm{ON}$ responses in patients with mutations in the GRM6 gene encoding mGluR6. Proc Natl Acad Sci USA 2005;102:4884-4889.

10 Weleber RG: The effect of age on human cone and rod ganzfeld electroretinograms. Invest Ophthalmol Vis Sci 1981;20:392-399.

11 Nakamura M, Ito S, Piao CH, Terasaki H, Miyake Y: Retinal and optic disc atrophy associated with a CACNA1F mutation in a Japanese family. Arch Ophthalmol 2003;121:1028-1033.

-12 Nakamura M, Ito S, Terasaki H, Miyake Y: Incomplete congenital stationary night blindness associated with symmetrical retinal atrophy. Am J Ophthalmol 2002;13:463-465. 


\section{Case Reports in Ophthalmology}



b

normal patient
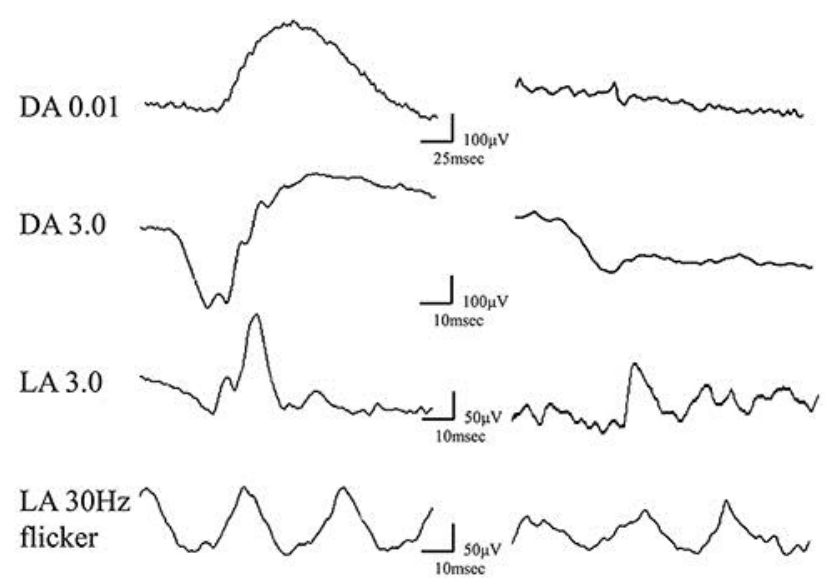

Fig. 1. Electroretinograms (ERGs) of the patient presented in this case report. a Single bright-flash, mixed rod-cone ERG elicited by a 20 -J white flash stimulus after $20 \mathrm{~min}$ of dark adaption. This showed a negative configuration and an unremarkable oscillatory potential. b ERGs performed according to the International Society for Clinical Electrophysiology of Vision protocol when the patient was 38 years of age. The rod response was non-recordable and the cone response was within normal range. DA, dark adaptation; LA, light adaptation. 


\section{Case Reports in Ophthalmology}
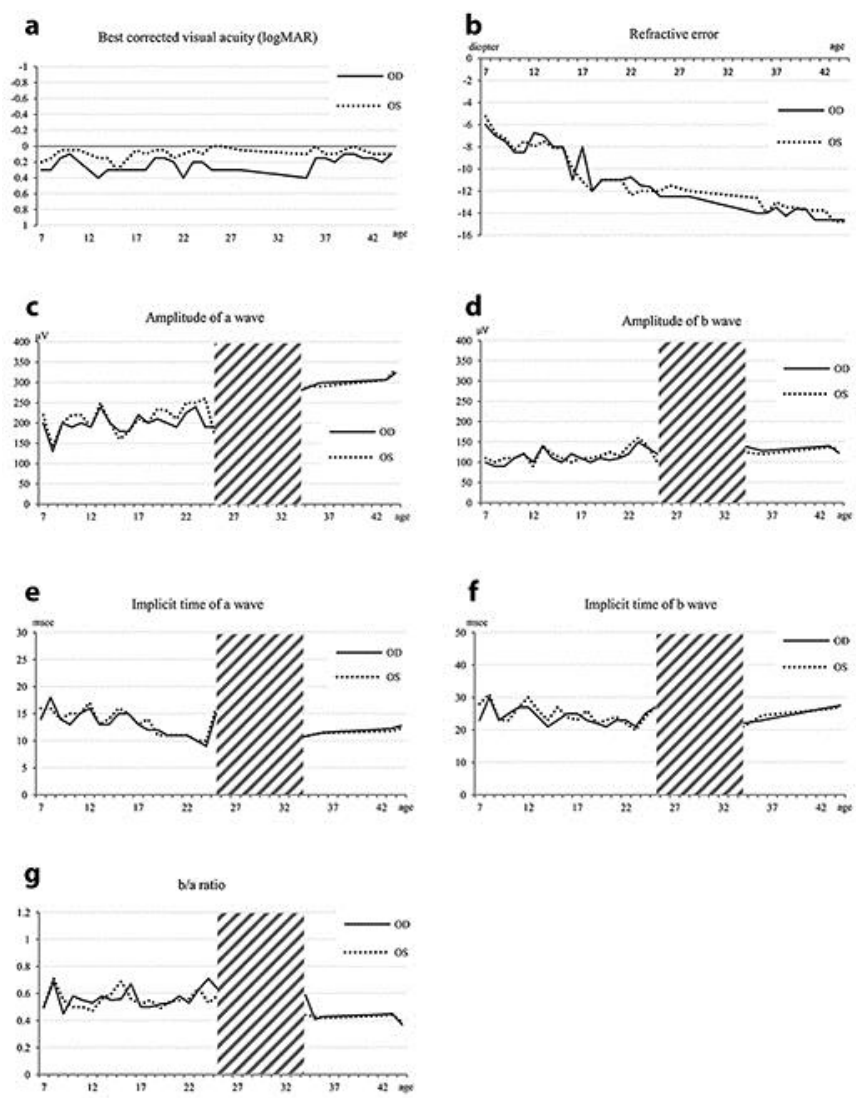

Fig. 2. Clinical course of the patient presented in this report. a Visual acuity. b Refraction. c Amplitude of the a-wave. $\mathbf{d}$ Amplitude of the $\mathbf{b}$-wave. e Implicit time of the a-wave. $\mathbf{f}$ Implicit time of the $\mathbf{b}$-wave. $\mathbf{g} \mathbf{b} / \mathbf{a}$ wave ratio. The oblique line shows the time when the patient resumed attending our hospital. 


\section{Case Reports in Ophthalmology}
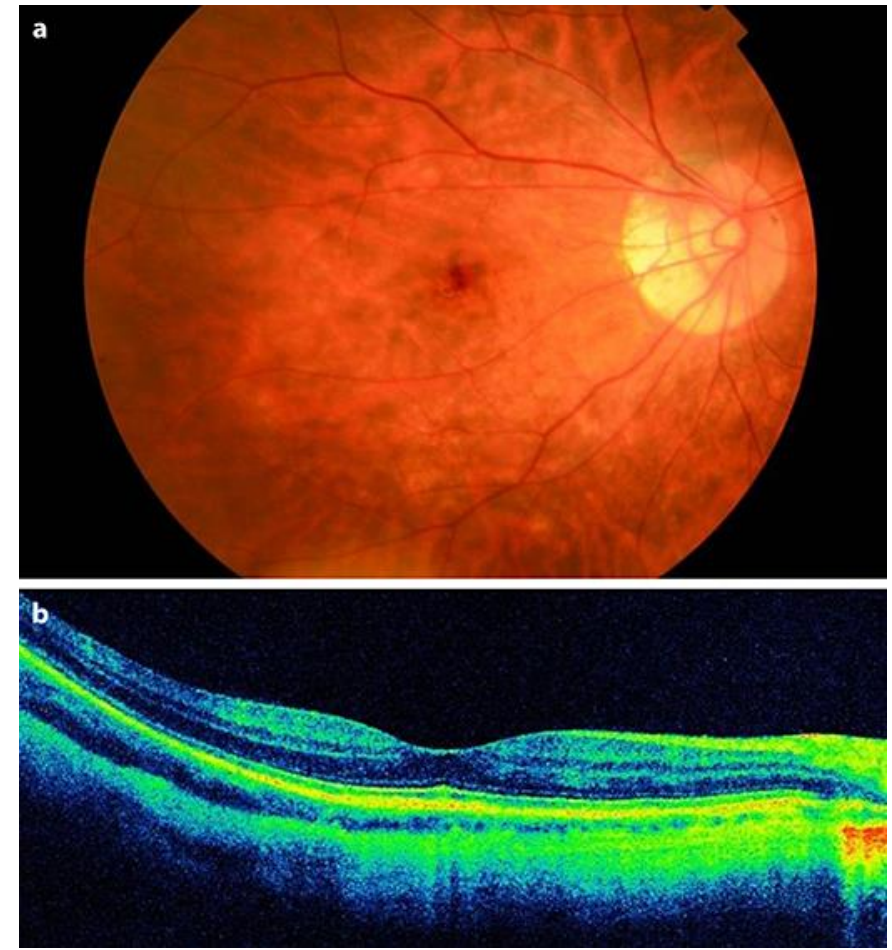

Fig. 3. Color fundus photograph and optical coherence tomography (OCT) image of the patient at present. a The fundus photograph of the right eye shows myopic changes but no atrophic changes. $\mathbf{b}$ The OCT image of the right eye shows a normal central retinal thickness and retinal lamination.

Kurata et al.: Long-Term Clinical Course in a Patient with Complete Congenital Stationary Night Blindness 